\title{
Efficacy and Safety of the Association of Nimodipine and Choline Alphoscerate in the Treatment of Cognitive Impairment in Patients with Cerebral Small Vessel Disease. The CONIVaD Trial
}

\author{
Emilia Salvadori ${ }^{1,2} \cdot$ Anna Poggesi ${ }^{1,2} \cdot$ Ida Donnini $^{1} \cdot$ Valentina Rinnoci $^{1} \cdot$ Guido Chiti $^{2,3} \cdot$ Martina Squitieri $^{2,4}$. \\ Laura Tudisco $^{2} \cdot$ Fabio Fierini $^{5}$. Anna Melone ${ }^{2} \cdot$ Francesca Pescini $^{6} \cdot$ Leonardo Pantoni $^{7}$ (1)
}

Accepted: 28 February 2021 / Published online: 15 April 2021

(c) The Author(s) 2021

\begin{abstract} vessel disease (SVD). mild-to-moderate cognitive impairment. was measured by the count of medicine bottles returned by patients.

Trial Registration Clinical Trial NCT03228498. Registered 25 July 2017.

\section{Introduction}

Vascular cognitive impairment (VCI) encompasses a wide range of possible etiologies, among which cerebral small vessel disease (SVD) is one of the most relevant due to its impact in terms of social and economic burden [1-3]. Several efforts have been made to identify a drug effective in VCI, but evidence is inconclusive, and at present, no approved pharmacological treatment exists $[4,5]$.
\end{abstract}

Background No approved treatment is available for patients with vascular cognitive impairment (VCI) due to cerebral small

Objective The CONIVaD (Choline Alphoscerate and Nimodipine in Vascular Dementia) study aimed to investigate the feasibility, efficacy, and safety of a combined treatment with choline alphoscerate and nimodipine in patients with SVD and

Methods Within this pilot, single-center (university hospital), double-blinded, randomized clinical trial, patients were randomized to two arms: 1-year treatment with nimodipine $30 \mathrm{mg}$ three times a day (TID) plus choline alphoscerate $600 \mathrm{mg}$ twice a day (BID) (arm 1) or nimodipine $30 \mathrm{mg}$ TID plus placebo BID (arm 2). Patients underwent an evaluation at baseline and after 12 months. Cognitive decline, defined as a $\geq 2$-point loss on the Montreal Cognitive Assessment, was the primary endpoint. Functional, quality of life, other cognitive measures, and safety were secondary endpoints. Treatment adherence

Results Sixty-two patients were randomized (31 each arm). Fourteen patients (22\%) dropped out for reasons including consent withdrawal $(n=9)$, adverse reactions $(n=4)$, and stroke $(n=1)$. Forty-eight patients (mean \pm SD age $75.1 \pm 6.8$ years), well balanced between arms, completed the study. Regarding adherence, of the prescribed total drug dose, $>75 \%$ was taken by $96 \%$ of patients for choline alphoscerate, $87.5 \%$ for placebo, and $15 \%$ for nimodipine. No statistically significant differences were found between the treatment groups for the primary cognitive outcome, nor for the secondary outcomes. Eight patients had non-serious adverse reactions; five presented adverse events.

Conclusion Patients' adherence to treatment was low. With this limitation, the combined choline alphoscerate-nimodipine treatment showed no significant effect in our cohort of VCI patients with SVD. The safety profile was good overall.

Leonardo Pantoni

leonardo.pantoni@unimi.it

Extended author information available on the last page of the article

\section{Key Points}

The association of choline alphoscerate and nimodipine showed no efficacy in improving cognitive abilities in patients with cerebral small vessel disease.

Treatment safety was adequate, while patients' adherence was not optimal, thus reducing the rate of patients treated as per protocol.

Further efforts are needed to find a specific treatment for patients with cognitive impairment and cerebral small vessel disease. 
Acetylcholinesterase inhibitors, approved for the treatment of Alzheimer's disease, have been tested in VCI patients according to the hypothesis of a functional disconnection in subcortical-cortical pathways, and thus in the cholinergic cortical network [6]. In the several controlled trials on the efficacy of acetylcholinesterase inhibitors in VCI patients, an overall positive effect on cognitive functions was found, but the treatment's efficacy regarding functional and global status was limited or absent [7-17]. Other cholinergic drugs, such as the choline precursors cytidine diphosphatecholine and choline alphoscerate (semisynthetic derivative of phosphate choline), have been tested in VCI patients, showing some positive effects on cognitive performances and a good tolerability profile [18-21].

Also, the vasoactive and neuroprotective mechanisms possibly induced by the dihydropyridinic calcium-antagonist nimodipine have been tested in VCI, with preliminary evidence of some positive effects on cognitive functions, good tolerability, and few side effects [22-29].

Taking into account the overall evidence from pharmacological studies, the statement of the American Heart Association/American Stroke Association (AHA/ASA) on VCI highlighted the need for further clinical trials designed according to a homogeneous case selection and an outcomes definition based on measures sensitive to executive function [17].

Considering the previous evidence of some beneficial cognitive effects of choline alphoscerate and nimodipine, we tested the hypothesis that a combined treatment with these two drugs could act synergistically on different targets, and thus induce a superior effect in comparison with single-treatment with nimodipine in patients affected by VCI related to SVD. We thus designed a pilot trial in patients with SVD and mild-to-moderate cognitive impairment with the aim of investigating the (1) feasibility and patient adherence, (2) efficacy, and (3) safety of the combined treatment.

\section{Methods}

The CONIVaD (Choline Alphoscerate and Nimodipine in Vascular Dementia) study is a 2-year, single-center, double-blinded, randomized clinical trial carried out at Careggi University Hospital in Florence, Italy. Its rationale and methodology, including sample size and statistical power estimation, have been extensively reported elsewhere [30].

The authors assert that all procedures contributing to this work comply with the ethical standards of the relevant national and institutional committees on human experimentation and with the Helsinki Declaration of 1975, as revised in 2008. The study was approved by the local ethics committee, and each patient provided written informed consent. The study is registered in the ClinicalTrials.gov registry (identifier number NCT03228498, registration date 25 July 2017).

Included patients were diagnosed as affected by cognitive impairment and SVD according to the following criteria: (1) cognitive impairment of mild-to-moderate degree defined by a Clinical Dementia Rating (CDR) score ranging between 0.5 and 2.0 [31] and (2) white matter hyperintensities (WMH) on brain magnetic resonance imaging (MRI) of moderate or severe degree according to the modified Fazekas scale (score 2 or 3 ) or moderate to severe white matter hypodensities on computed tomography according to van Swieten scale (score $>2$ ) [32, 33]. When MRI was available, the degree of WMH severity was rated on fluid attenuated inversion recovery (FLAIR) sequences, taking into account only deep and subcortical white matter lesions. The modified Fazekas scale is a visual scale based on three severity classes: grade $1($ mild $\mathrm{WMH})=$ single lesions smaller than $10 \mathrm{~mm}$, areas of 'grouped' lesions smaller than $20 \mathrm{~mm}$ in diameter; grade 2 (moderate $\mathrm{WMH})=$ single lesions between 10 and $20 \mathrm{~mm}$, areas of 'grouped' lesions more than $20 \mathrm{~mm}$ in diameter, no more than 'connecting bridges' between individual lesions; and grade 3 (severe WMH) = single lesions or confluent areas of hyperintensity $20 \mathrm{~mm}$ or more in diameter [32].

Exclusion criteria were as follows: (1) diagnosis of major depression, schizophrenia, major anxiety syndrome, or bipolar disorder; (2) diagnosis of cognitive impairment related to Alzheimer's disease, Parkinson's disease, Huntington's disease, and frontotemporal lobar degeneration based on clinical and neuroimaging findings in line with the standard diagnostic criteria [34, 35]; (3) diagnosis of cognitive impairment of other origin (i.e., vitamin $B_{12}$ and folic acid deficiency, thyroid disorders, metabolic diseases, head trauma, tumor or infections of the central nervous system, normal pressure hydrocephalus); (4) medical conditions expected to progress, recur, or change to such a degree as to interfere with the assessment of the clinical and mental status; (5) vision, hearing, or language impairments severe enough to interfere with cognitive assessment; (6) clinically relevant heart failure, respiratory insufficiency, electrocardiographic abnormalities (bradycardia or tachycardia); (7) myocardial infarction within the past 6 months; (8) stroke still requiring neurological rehabilitation; (9) severe/ untreated hypertension; (10) clinically relevant liver function impairment; (11) insulin-dependent diabetes mellitus; (12) idiopathic epilepsy and anti-epileptic treatment; (13) severe anemia, gastrointestinal disease, or renal failure; (14) cancer; (15) known intolerance to study drugs; and (16) expected poor compliance or coexistent serious illnesses that would likely imply the patient would drop out before the end of the trial.

At baseline, according to the study protocol, each patient underwent an extensive clinical (medical history, standard 
cardiovascular parameters, and neurological examination), functional, quality-of-life, mood, and neuropsychological assessment [30].

After baseline assessment, participants were randomly assigned to one of the two arms of treatment: (1) oral nimodipine $30 \mathrm{mg}$ three times a day (TID) plus oral choline alphoscerate $600 \mathrm{mg}$ twice a day (BID) (arm 1) and (2) nimodipine $30 \mathrm{mg}$ TID plus placebo BID ( $\operatorname{arm} 2$ ). Both drugs were administered as oral solutions: nimodipine was taken by means of a dropper bottle in the morning, early afternoon, and evening at least $1 \mathrm{~h}$ before or $2 \mathrm{~h}$ after meals, while choline alphoscerate was taken by means of a phial in the morning and in the afternoon.

Treatment was designed to last for 12 months. The study protocol comprised two follow-up visits: (1) at 6 months, with evaluation of standard cardiovascular parameters, side effects, routine blood tests, and compliance with and adherence to treatment, and (2) at 12 months, with evaluation of side effects and compliance with and adherence to treatment and an extensive clinical and neuropsychological assessment performed according to the baseline protocol.

The randomization list was generated prior to the enrollment start using the procedure PROC PLAN of SAS 9.4 for Windows by the statistician of the pharmaceutical company providing the drugs. The statistician was blind to patients' characteristics. In order to cover any potential loss of product for any given reason during the trial, the randomization list was stratified into 12 blocks of six treatment kits each, for a total of 72 treatment kits. The principal investigator and the relevant personnel involved in the study were blinded to the allocation until the end of study, thus allowing a doubleblind trial to be conducted.

\subsection{Outcome Measures}

\subsubsection{Primary Outcome}

The primary outcome was the reduction of cognitive decline, defined as the loss of $\geq 2$ points on the Montreal Cognitive Assessment (MoCA) [36] test 12 months after baseline, in patients treated with the combination of oral nimodipine and choline alphoscerate (arm 1) compared to patients treated with the combination of nimodipine and placebo ( $\operatorname{arm} 2)$.

\subsubsection{Secondary Outcomes}

Secondary outcomes included the effects of treatments on:

1. Functional status measured by means of each of three scales administered to the caregiver: the Activities of Daily Living (ADL) scale (score range 0-6; higher scores represent less disability), Instrumental Activities of Daily Living (IADL) scale (score range 0-8; higher scores represent more disability), and Disability Assessment in Dementia scale (total score as a percentage; higher scores represent less disability) [37-39].

2. Mood and quality of life measured respectively by means of two questionnaires administered to the patient: the Stroke Adapted-Sickness Impact Profile 30 (SASIP30) (total score as a percentage; higher scores represent worse health status) and Center for Epidemiologic Studies Depression Scale (CES-D) (score range 0-60; higher scores represent greater presence of depressive symptoms) [40, 41].

3. Cognitive performance on neuropsychological tests evaluating the following: maintained attention (Symbol Digit Modalities Test; score range 0-110; higher scores represent better performance), selective attention (Color Word Stroop Test; total score is time to complete; higher scores represent worse performance), psychomotor speed and divided attention (Trail Making Test parts $\mathrm{A}$ and $\mathrm{B}$; total score is time to complete; higher scores represent worse performance), and memory (Rey Auditory-Verbal Learning Test immediate and delayed recall; score ranges $0-75$ and $0-15$, respectively; higher scores represent better performance) [42].

The tolerability of treatment and the incidence of adverse reactions and events were also studied. The distinction between adverse reactions and adverse events was based on the fact that a causal relationship was suspected for the former, but was not required for the latter [43].

Treatment adherence was measured indirectly by the count of medicine bottles returned by patients, i.e., the number of empty phials for choline alphoscerate and the decrease in drug solution in dropper bottles for nimodipine. Adherence to treatment was evaluated in patients who completed the study, taking into account the total doses of choline alphoscerate, placebo, or nimodipine taken in 12 months and dividing patients into four categories according to the ratio between the taken and the theoretical total dose of treatment: $\leq 25 \%, 26-50 \%, 51-75 \%$, and $>75 \%$.

\subsection{Statistical Analysis}

Sample size estimation was determined a priori based on the MoCA performances in a sample of patients with SVD and mild cognitive impairment who had been evaluated longitudinally in the observational V-MCI Tuscany study [44]. Taking into account the five-point standard deviation of the MoCA score at 12 months and the correlation between baseline and follow-up MoCA scores equivalent to 0.70, for a treatment-arm assignment equivalent to $1: 1$, a sample size of 60 patients was estimated to guarantee $80 \%$ equal power to the study in comparison to the alternative hypothesis, with 
a $20 \%$ significance level according to the exploratory nature of this study.

Descriptive analyses were used to illustrate the clinical characteristics and the distributions of the rates of adherence to the treatments and of adverse reactions and events. To search for possible systematic biases either in patients' withdrawal of consent or treatment allocation, independent sample $t$ tests and chi-square tests were used to compare baseline characteristics between patients who completed the study and those who dropped out, and between the two treatment arms.

The MoCA score changes were analyzed according to different approaches:

- Rates of patients presenting a loss of $\geq 2$ points on the MoCA test between baseline and 12-month visits were computed and then compared between the treatment groups using a chi-square test (primary outcome).

- Repeated measures factorial analysis of variance (ANOVA): Introducing into the statistical model the MoCA test scores at baseline and at 12 months as dependent variables and the arm of treatment as independent variable.

- Changes in scores $(\Delta)$ approach: MoCA delta scores $(\Delta \mathrm{s})$ were calculated by computing the difference between baseline and 12-month scores for each patient (a positive score indicated an improvement). Delta scores were analyzed using independent sample $t$ tests, with treatment as an independent variable.

- Clinical significance approach: After the classification of each patient's performance as 'normal,' 'borderline,' or 'abnormal' at each visit according to national norms, variations in performance categories between baseline and 12 months were dichotomized as 'stable or better' or 'worst.' Variations in performance categories were analyzed using Chi-square tests.

The secondary cognitive and functional outcomes have been analyzed according to the repeated measures factorial ANOVA and delta scores approaches. The clinical significance approach was further used only for cognitive tests.

\section{Results}

Enrolment started on May 2, 2017 and was completed on July 23, 2018.

Patient selection and attrition from the screening phase to follow-up assessments are shown in Fig. 1. Among the 263 screened patients, 201 (76\%) were excluded from the study. Main reasons for exclusion were refusal $(n=42)$, not fitting the cognitive criteria ( $n=41$ normal, $n=24$ demented), expected low compliance $(n=29)$, functional deficit or clinical complications $(n=24)$, and not fitting the neuroimaging criteria $(n=15)$. Sixty-two Caucasian patients (24\%) [30 males (48\%), mean ( \pm SD) age and years of education $75.8 \pm 7$ and $8.3 \pm 4.6$, respectively] were finally enrolled. As shown in Table 1, the vascular risk factors distribution and the cognitive and functional test profiles were those expected in a sample of patients with SVD and cognitive impairment of mild-to-moderate degree. Out of the 62 enrolled patients, 31 were randomized to arm 1 (combination of nimodipine and choline alphoscerate) and 31 to arm 2 (combination of nimodipine and placebo).

The rate of drop-out among the 62 enrolled patients was $22 \%$ ( $n=14 ; 12$ occurred within the first 3 months, and two occurred between the 6th and 9th month), and was equally balanced between the two arms (Fig. 1 and Table 1). Reasons for drop-out were treatment interruption due to consent withdrawal ( $n=4$ in $\operatorname{arm} 2, n=5$ in arm 1) or symptoms compatible with an adverse reaction or event $(n=3$ in arm $2, n=2$ in arm 1) (Fig. 1).

Comparison between patients who completed the study and those who dropped out showed no statistically significant differences in baseline characteristics, except for education level, which was significantly higher in patients who completed the study (Table 1).

The final study cohort included 48 patients well balanced between the two treatment groups. As shown in Table 2, the two treatment arms had no significant differences in baseline characteristics, except for education, which was significantly higher in arm 1.

Distributions of adherence to the treatments are shown in Fig. 2. Among the 48 patients who completed the study, considering the total doses of drug taken in 12 months, an adherence level of $>75 \%$ was reached by 23 patients (96\%) for choline alphoscerate (arm 1), 21 patients (87.5\%) for placebo (arm 2), and only by seven patients (15\%) for nimodipine (arm 1 and arm 2) (Fig. 2).

Data on the primary cognitive outcome are shown in Table 3. No statistically significant difference was found between the treatment groups in performances on the MoCA test in any of the different statistical approaches. Also, comparisons for the secondary cognitive, functional, and mood and quality-of-life outcomes did not show any statistically significant difference between the treatment arms (Table 3 ).

Distributions of adverse reactions and events in the baseline sample are shown in Table 4. Eight patients referred a total of 14 symptoms compatible with an adverse reaction, of which 13 fell within those known for the drugs; none was classified as serious; five were referred from two patients in arm 1 (one patient interrupted the treatment) and nine from six patients in arm 2 (three patients interrupted the treatment and one decided to decrease the treatment dose). The total number of adverse events was five (two in arm 1 and three in arm 2); four of them were classified as serious, 


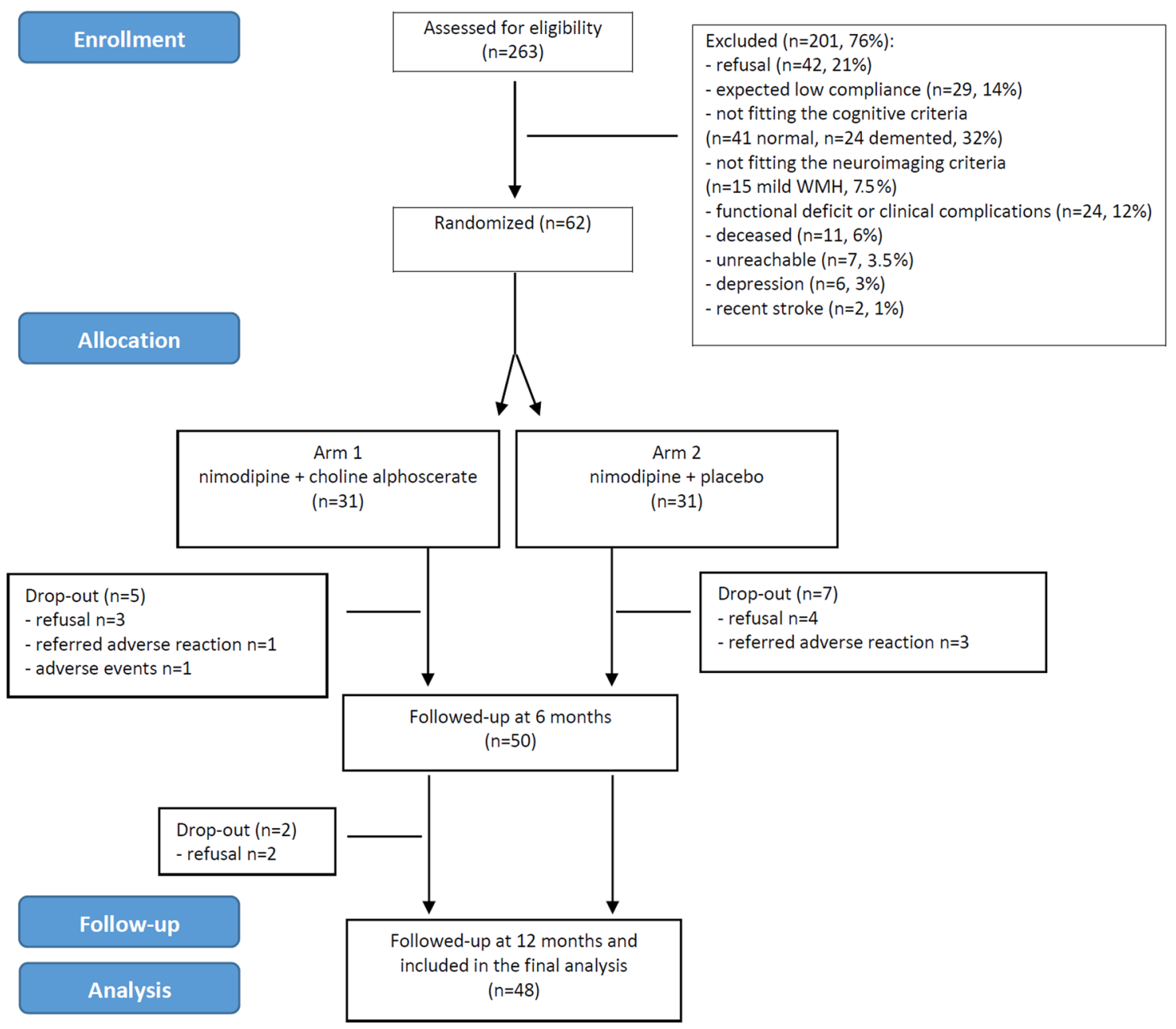

Fig. 1 Patients' selection and attrition from the screening phase to follow-up assessments (CONSORT flow diagram). CONSORT Consolidated Standards of Reporting Trials, $W M H$ white matter hyperintensities

and one patient in arm 1 interrupted the treatment (cerebral hemorrhage).

\section{Discussion}

In the CONIVaD trial, we were not able to show any efficacy of the association of choline alphoscerate and nimodipine in improving cognition in patients with cognitive impairment and SVD. No safety or tolerability concerns were identified, but the trial also generates some considerations regarding the adherence of patients to the proposed treatment approach. In particular, the taking of nimodipine represented an issue in our pilot experience because only a very small proportion of patients took the prescribed dosage. As a consequence, only a few patients could be considered as really having been treated as per protocol. At present, we have no clear explanation for this.
We can hypothesize that one reason was the trial protocol requirement of three doses a day, which was derived from the typical regimen for nimodipine, while choline alphoscerate requires two doses a day. Moreover, administration via dropper and the requirement of avoiding mealtimes could have further reduced patients' adherence. Another possible issue is the fact that the trial was set to last for 12 months, a duration longer than that of many other trials, which was deliberately chosen because chronic therapies in cognitively impaired patients should be tested using a long-term perspective, both in terms of efficacy and adherence to treatment. Furthermore, the presence of a high early drop-out rate suggests that future studies using similar approaches should include a run-in period.

At present, therefore, VCI remains a condition lacking specific treatment, and this implies that further efforts are needed. Whether better adherence to the complete 
Table 1 Comparison of baseline characteristics in total sample, patients who completed the study, or dropped out

\begin{tabular}{|c|c|c|c|c|c|}
\hline & Score range & $\begin{array}{l}\text { Total sample } \\
n=62\end{array}$ & $\begin{array}{l}\text { Study completed } \\
n=48\end{array}$ & $\begin{array}{l}\text { Drop-out } \\
n=14\end{array}$ & $P$ \\
\hline Age, years & - & $75.8 \pm 7$ & $75.7 \pm 6.8$ & $76.4 \pm 9.2$ & $0.725 *$ \\
\hline Education, years & - & $8.3 \pm 4.6$ & $7.7 \pm 3.9$ & $10.4 \pm 5.9$ & $0.047 *$ \\
\hline Sex (\% males) & - & $48 \%$ & $48 \%$ & $50 \%$ & $0.891^{\#}$ \\
\hline Hypertension & - & $74 \%$ & $71 \%$ & $86 \%$ & $0.263^{\#}$ \\
\hline Hypercholesterolemia & - & $81 \%$ & $85 \%$ & $64 \%$ & $0.078^{\#}$ \\
\hline Diabetes & - & $21 \%$ & $21 \%$ & $21 \%$ & $0.962^{\#}$ \\
\hline Smoking habits & - & $43 \%$ & $40 \%$ & $57 \%$ & $0.244^{\#}$ \\
\hline History of stroke & - & $42 \%$ & $40 \%$ & $50 \%$ & $0.487^{\#}$ \\
\hline Alcohol consumption & - & $61 \%$ & $58 \%$ & $71 \%$ & $0.376^{\#}$ \\
\hline Physical activity & - & $50 \%$ & $54 \%$ & $36 \%$ & $0.224^{\#}$ \\
\hline MoCA score & $0-30$ & $18.7 \pm 4.3$ & $19 \pm 4$ & $17.5 \pm 5.2$ & $0.264 *$ \\
\hline ADL (preserved items) & $0-6$ & $5.6 \pm 0.9$ & $5.7 \pm 0.8$ & $5.5 \pm 1.2$ & $0.501 *$ \\
\hline IADL (impaired items) & $0-8$ & $3.2 \pm 2.8$ & $3.0 \pm 2.7$ & $3.7 \pm 3.1$ & $0.382 *$ \\
\hline DAD score & $0-100$ & $84.7 \pm 21$ & $85.4 \pm 20.0$ & $82.4 \pm 24.9$ & $0.637 *$ \\
\hline Combined treatment (\%) & - & - & $50 \%$ & $50 \%$ & $1.000^{\#}$ \\
\hline
\end{tabular}

Bold values denote statistical significance at the $p<0.05$ level

$A D L$ Activities of Daily Living, $D A D$ Disability Assessment in Dementia, IADL Instrumental Activities of Daily Living, MoCA Montreal Cognitive Assessment

*Independent sample $t$ tests

${ }^{\#}$ Chi square tests

protocol, perhaps reached with a less demanding regimen, would have generated a different outcome is an open question.

Within the VCI spectrum, in the present study, SVD was defined by evidence of moderate to severe white matter lesions to guarantee adequate homogeneity of the study sample, despite possibly reducing generalizability and sample size, in line with previous experiences gathered by the authors both in the LADIS European collaboration and in the V-MCI Tuscany study [44, 45].

Our study has limitations. The first one is the small sample size. However, we could not identify any trend in the results, and therefore, it seems unlikely that the same protocol could lead to different results in a larger sample. Based on this pilot experience, it can be hardly hypothesized that a large, multicentric trial could lead to different results, unless better adherence is achieved, for example, by reducing the number of daily treatment doses from three to two. Another limitation is the lack of a placebo arm. Since nimodipine is not a licensed treatment for VCI, the inclusion of a placebo arm could have been appropriate. The comparison treatment nimodipine-placebo has already been tested and proven to be of limited efficacy, and thus, we wanted to explore the role of a twodrug treatment [25]. Within this study design, a two-bytwo factorial analysis would have allowed comparisons of the drug combination against either drug alone or double placebo, thus increasing our potential to explore the synergistic effect. Despite the added value of a more complex study design, feasibility issues and the foreseen sample size forced us to limit our pilot study to two arms. A third possible limitation is the lack of an intention-to-treat (ITT) analysis, which could have minimized the effect of noncompliant patients and dropouts. However, in this small pilot trial, the cognitive assessment at the time of drop-out was not foreseen, and thus an ITT analysis was not applicable. Another limitation is the fact that we used drugs that had been already tested singularly in patients with VCI; the novelty of this trial approach was the assessment of the possible use of a combination of these drugs. A final possible limitation is the choice of the cholinergic drug for this trial; in the future, it could be interesting to explore whether different results could be achieved with an acetylcholinesterase inhibitor added to the calcium antagonist.

\section{Conclusions}

In the CONIVaD pilot study, the combined treatment with choline alphoscerate and nimodipine in patients with SVD and mild-to-moderate cognitive impairment resulted in low patient adherence to treatment, particularly for nimodipine, and no significant efficacy. Safety 
Table 2 Comparisons of baseline characteristics of patients in the two treatment arms

\begin{tabular}{|c|c|c|c|c|}
\hline & Score range & $\begin{array}{l}\text { Arm } 1 \\
n=24\end{array}$ & $\begin{array}{l}\text { Arm } 2 \\
n=24\end{array}$ & $p$ \\
\hline Age, years & - & $76.3 \pm 5.5$ & $75 \pm 7.2$ & $0.475^{*}$ \\
\hline Education, years & - & $9.3 \pm 4.2$ & $6 \pm 2.9$ & $0.003^{*}$ \\
\hline Sex (\% males $)$ & - & $42 \%$ & $62 \%$ & $0.149^{\#}$ \\
\hline Hypertension & - & $58 \%$ & $83 \%$ & $0.057^{\#}$ \\
\hline Hypercholesterolemia & - & $87 \%$ & $83 \%$ & $0.683^{\#}$ \\
\hline Diabetes & - & $21 \%$ & $21 \%$ & $1.000^{\#}$ \\
\hline Smoking habits & - & $42 \%$ & $37 \%$ & $0.768^{\#}$ \\
\hline History of stroke & - & $37 \%$ & $42 \%$ & $0.768^{\#}$ \\
\hline Alcohol consumption & - & $58 \%$ & $58 \%$ & $1.000^{\#}$ \\
\hline Physical activity & - & $50 \%$ & $58 \%$ & $0.562^{\#}$ \\
\hline MoCA score & $0-30$ & $19.9 \pm 3.2$ & $18.1 \pm 4.6$ & $0.121 *$ \\
\hline RAVL score (immediate) & $0-75$ & $32.7 \pm 7.2$ & $35.8 \pm 9.4$ & $0.214^{*}$ \\
\hline RAVL score (recall) & $0-15$ & $6.3 \pm 2.5$ & $6.9 \pm 3$ & $0.415^{*}$ \\
\hline Symbol Digit Modalities Test score & $0-110$ & $35.5 \pm 8.3$ & $37.3 \pm 8.5$ & $0.504 *$ \\
\hline Color Word Stroop Test & Time (s) & $41.3 \pm 47.1$ & $36.5 \pm 26.5$ & $0.670 *$ \\
\hline Trail Making Test part A & Time (s) & $67.9 \pm 61.1$ & $58.6 \pm 44.9$ & $0.571 *$ \\
\hline Trail Making Test part B & Time (s) & $121 \pm 54.5$ & $113.9 \pm 70.8$ & $0.835^{*}$ \\
\hline ADL (preserved items) & $0-6$ & $5.7 \pm 0.9$ & $5.7 \pm 0.7$ & $0.864 *$ \\
\hline IADL (impaired items) & $0-8$ & $3.2 \pm 2.9$ & $2.8 \pm 2.5$ & $0.598^{*}$ \\
\hline DAD score & $0-100$ & $84.3 \pm 18.4$ & $86.5 \pm 21.8$ & $0.707 *$ \\
\hline CES-D score & $0-60$ & $14.7 \pm 10.7$ & $13.3 \pm 10.4$ & $0.654 *$ \\
\hline SA-SIP30 score & $0-100$ & $32.3 \pm 22.8$ & $27.2 \pm 20.7$ & $0.416^{*}$ \\
\hline
\end{tabular}

Bold values denote statistical significance at the $p<0.05$ level

$A D L$ Activities of Daily Living, CES-D Center for Epidemiologic Studies Depression Scale, DAD Disability Assessment in Dementia, IADL Instrumental Activities of Daily Living, MoCA Montreal Cognitive Assessment, RAVL Rey Auditory-Verbal Learning Test, SA-SIP30 Stroke Adapted-Sickness Impact Profile 30

*Independent sample $t$ tests

${ }^{\#}$ Chi square tests

Fig. 2 Adherence to the treatment (expressed as the ratio between the taken dose and the theoretical total dose) in patients who completed the study $(n=48)$

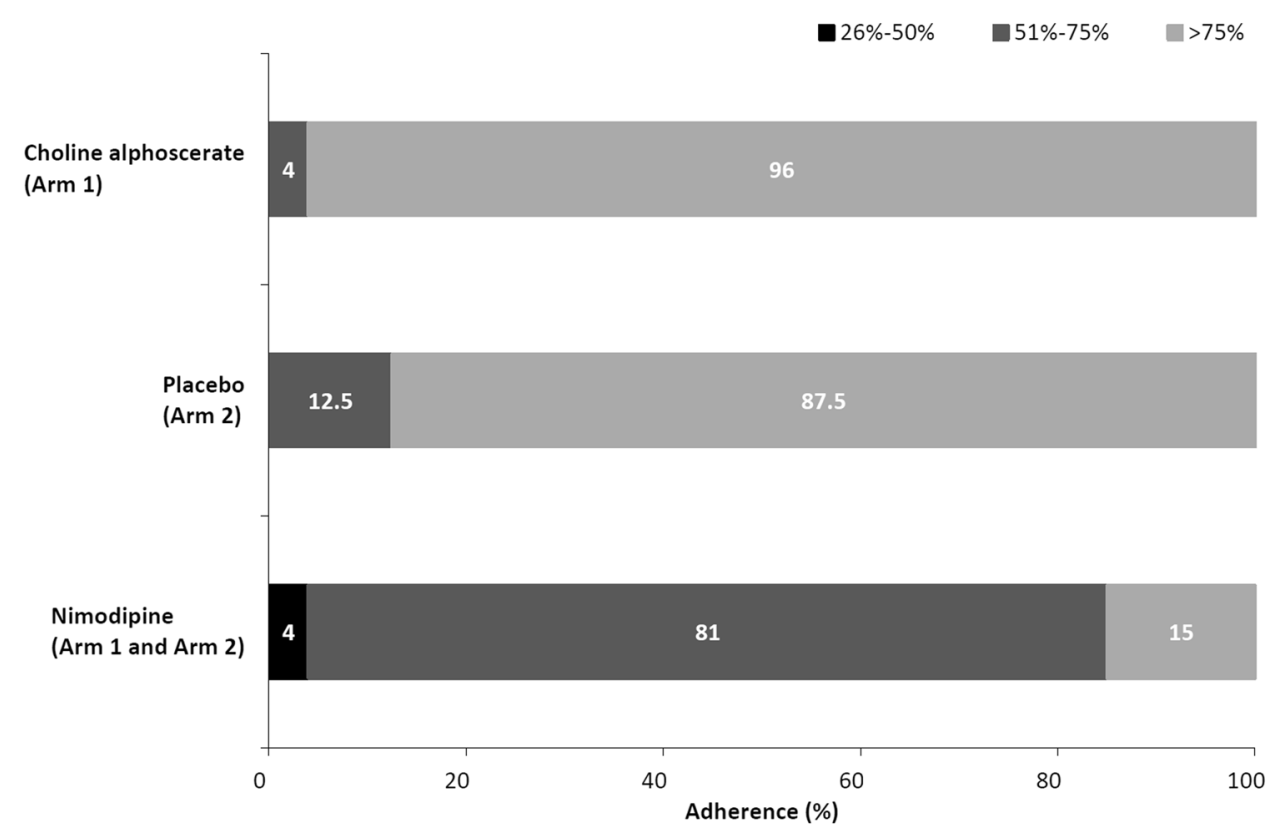


Table 3 Comparisons between arms of treatment for the primary cognitive outcome (MoCA) and for the secondary cognitive and functional outcomes

\begin{tabular}{|c|c|c|c|c|c|c|}
\hline & \multirow[t]{2}{*}{ Score range } & \multicolumn{2}{|l|}{$\begin{array}{l}\text { Arm } 1 \\
n=24\end{array}$} & \multicolumn{2}{|l|}{$\begin{array}{l}\text { Arm 2 } \\
n=24\end{array}$} & \multirow[t]{2}{*}{$P$} \\
\hline & & Baseline & 12 months & Baseline & 12 months & \\
\hline \multicolumn{7}{|l|}{ MoCA } \\
\hline Loss $\geq 2$ points & & $7(29 \%)$ & & $7(29 \%)$ & & 1.000 \\
\hline Adjusted score & $0-30$ & $19.9 \pm 3.2$ & $19.1 \pm 3.8$ & $18.1 \pm 4.6$ & $18.2 \pm 5.1$ & 0.232 \\
\hline$\Delta \mathrm{s}^{\#}$ & & $-0.82 \pm 2.5$ & & $0.05 \pm 3.7$ & & 0.342 \\
\hline \multicolumn{7}{|l|}{ RAVL (immediate) } \\
\hline Stable/better* & & $58 \%$ & & $79 \%$ & & 0.119 \\
\hline Adjusted score & $0-75$ & $32.7 \pm 7.2$ & $35.6 \pm 9.8$ & $35.8 \pm 9.4$ & $37.0 \pm 8.5$ & 0.352 \\
\hline$\Delta \mathrm{s}^{\#}$ & & $2.9 \pm 5.5$ & & $1.3 \pm 7.4$ & & 0.378 \\
\hline \multicolumn{7}{|l|}{ RAVL (recall) } \\
\hline Stable/better* & & $83 \%$ & & $79 \%$ & & 0.712 \\
\hline Adjusted score & $0-15$ & $6.3 \pm 2.5$ & $6.6 \pm 3.0$ & $6.9 \pm 3.0$ & $7.4 \pm 2.7$ & 0.350 \\
\hline$\Delta \mathrm{s}^{\#}$ & & $0.3 \pm 1.7$ & & $0.4 \pm 2.2$ & & 0.830 \\
\hline \multicolumn{7}{|c|}{ Symbol Digit Modalities Test } \\
\hline Stable/better* & & $83 \%$ & & $79 \%$ & & 0.712 \\
\hline Adjusted score & $0-110$ & $35.5 \pm 8.3$ & $35.8 \pm 9.9$ & $37.3 \pm 8.5$ & $34.9 \pm 7.2$ & 0.861 \\
\hline$\Delta \mathrm{s}^{\#}$ & & $0.3 \pm 6.6$ & & $-2.4 \pm 7.3$ & & 0.223 \\
\hline \multicolumn{7}{|c|}{ Color Word Stroop Test } \\
\hline Stable/better* & & $85 \%$ & & $82 \%$ & & 0.782 \\
\hline Adjusted score & Time (s) & $41.3 \pm 47.1$ & $54.7 \pm 69.0$ & $36.3 \pm 27.1$ & $45 \pm 42.9$ & 0.579 \\
\hline$\Delta s^{\#}$ & & $-13.4 \pm 36.1$ & & $-8.7 \pm 44.5$ & & 0.694 \\
\hline \multicolumn{7}{|l|}{ TMT part A } \\
\hline Stable/better* & & $61 \%$ & & $70 \%$ & & 0.536 \\
\hline Adjusted score & Time (s) & $62.1 \pm 55.7$ & $70.1 \pm 51.9$ & $58.6 \pm 44.9$ & $70.8 \pm 35.5$ & 0.751 \\
\hline$\Delta \mathrm{s}^{\#}$ & & $-7.9 \pm 39.3$ & & $-12.1 \pm 46.6$ & & 0.751 \\
\hline \multicolumn{7}{|l|}{ TMT part B } \\
\hline Stable/better* & & $65 \%$ & & $56 \%$ & & 0.546 \\
\hline Adjusted score & Time (s) & $121 \pm 54.5$ & $95.7 \pm 37.1$ & $68.9 \pm 36.7$ & $78.9 \pm 66.1$ & 0.256 \\
\hline$\Delta \mathrm{s}^{\#}$ & & $25.3 \pm 38.2$ & & $-9.9 \pm 72.5$ & & 0.306 \\
\hline \multicolumn{7}{|l|}{ DAD } \\
\hline Stable/better* & & $95 \%$ & & $80 \%$ & & 0.185 \\
\hline Percentage score & $0-100$ & $83.6 \pm 18.5$ & $83.4 \pm 22.7$ & $86.5 \pm 21.8$ & $79.91 \pm 25.2$ & 0.957 \\
\hline$\Delta \mathrm{s}^{\#}$ & & $-0.19 \pm 12.2$ & & $-6.62 \pm 17.3$ & & 0.149 \\
\hline \multicolumn{7}{|l|}{ CES-D } \\
\hline Total score & $0-60$ & $14.7 \pm 10.7$ & $13.7 \pm 7.6$ & $13.3 \pm 10.4$ & $15.1 \pm 9.0$ & 10.000 \\
\hline$\Delta \mathrm{s}^{\#}$ & & $1 \pm 9.3$ & & $-1.7 \pm 8.4$ & & 0.289 \\
\hline \multicolumn{7}{|l|}{ SA-SIP30 } \\
\hline Percentage score & $0-100$ & $32.3 \pm 22.8$ & $27.3 \pm 22.9$ & $27.2 \pm 20.7$ & $28.6 \pm 18.9$ & 0.734 \\
\hline$\Delta \mathrm{s}^{\#}$ & & $5 \pm 18.4$ & & $-1.4 \pm 14.1$ & & 0.181 \\
\hline \multicolumn{7}{|l|}{ ADL } \\
\hline Preserved items & $0-6$ & $5.6 \pm 0.9$ & $5.4 \pm 1.3$ & $5.7 \pm 0.7$ & $5.5 \pm 1.2$ & 0.780 \\
\hline \multicolumn{7}{|l|}{ IADL } \\
\hline Impaired items & $0-8$ & $3.4 \pm 2.9$ & $3.3 \pm 3.2$ & $2.8 \pm 2.5$ & $3.0 \pm 2.6$ & 0.578 \\
\hline
\end{tabular}

$A D L$ Activities of Daily Living, CES-D Center for Epidemiologic Studies Depression Scale, DAD Disability Assessment in Dementia, IADL Instrumental Activities of Daily Living, MoCA Montreal Cognitive Assessment, RAVL Rey Auditory-Verbal Learning Test, SA-SIP30 Stroke Adapted-Sickness Impact Profile 30, TMT Trail Making Test

*Percentages of patients having a stable or better evaluation (clinical significance approach); Chi-square tests

${ }^{\#}$ A positive $\Delta$ score indicates improvement ( $\Delta s$ approach); independent sample $t$ tests 
Table 4 Adverse reactions and events

\begin{tabular}{|c|c|c|c|c|c|c|}
\hline \multirow[t]{2}{*}{ Expected } & \multirow[t]{2}{*}{ Serious } & \multirow[t]{2}{*}{ Total sample } & \multicolumn{2}{|l|}{ Arm 1} & \multicolumn{2}{|l|}{ Arm 2} \\
\hline & & & $\begin{array}{l}\text { Study } \\
\text { completed }\end{array}$ & Drop-out & $\begin{array}{l}\text { Study } \\
\text { completed }\end{array}$ & Drop-out \\
\hline
\end{tabular}

\begin{tabular}{|c|c|c|c|c|c|c|c|}
\hline \multicolumn{8}{|l|}{ Adverse reactions } \\
\hline Allergic reaction, rash & Yes & No & 3 & 0 & 1 & 1 & 1 \\
\hline Headache & Yes & No & 3 & 1 & 1 & 0 & 1 \\
\hline Tachycardia, bradycardia & Yes & No & 2 & 1 & 0 & 1 & 0 \\
\hline Hypotension, vasodilation, syncope, edema & Yes & No & 2 & 0 & 0 & 0 & 2 \\
\hline Nausea, constipation, diarrhea, flatulence & Yes & No & 3 & 0 & 0 & 1 & 2 \\
\hline Hypertension, agitation & No & No & 1 & 0 & 1 & 0 & 0 \\
\hline Total adverse reactions & & & 14 & 2 & 3 & 3 & 6 \\
\hline Trial drug decreased due to adverse reaction & & & 1 & 0 & 0 & 1 & 0 \\
\hline Withdrawn due to adverse reaction & & & 4 & 0 & 1 & 0 & 3 \\
\hline \multicolumn{8}{|l|}{ Adverse events } \\
\hline Cerebral hemorrhage & No & Yes & 1 & 0 & 1 & 0 & 0 \\
\hline Incidental carcinoma diagnosis & No & Yes & 3 & 0 & 0 & 3 & 0 \\
\hline Varicella zoster virus infection relapse & No & No & 1 & 1 & 0 & 0 & 0 \\
\hline Total adverse events & & & 5 & 1 & 1 & 3 & 0 \\
\hline Trial drug decreased due to adverse event & & & 0 & 0 & 0 & 0 & 0 \\
\hline Withdrawn due to adverse event & & & 1 & 0 & 1 & 0 & 0 \\
\hline
\end{tabular}

and tolerability were adequate. Further efforts are needed to find a specific treatment in patients with VCI.

\section{Declarations}

Funding Open access funding provided by Università degli Studi di Milano within the CRUI-CARE Agreement. MDM Spa Company (Monza, Italy) provided the active drugs and placebo as free donations. The company had no role in the trial's design or in carrying out the trial, and did not have any role in data analyses.

Conflict of interest AP, FP, ID, VR, GC, MS, LT, AM, and FF report no disclosures. LP is a member of the editorial boards of Cerebrovascular Diseases, Acta Neurologica Scandinavica, Neurological Sciences, Cerebral Circulation-Cognition and Behavior, and European Stroke Journal and a section editor (Vascular Cognitive Impairment) of Stroke. ES is a member of the editorial board of Cerebral Circulation-Cognition and Behavior.

Ethics approval and consent to participate The study was performed in accordance with the 1964 Helsinki Declaration and its later amendments and with the ethical standards of the institutional ethics committee, and was approved by the ethics committee of the Careggi University Hospital, Florence, Italy. Informed consent was obtained from all individual participants included in the study.

Consent to publish Patients signed informed consent regarding publishing their data.

Code availability Not applicable.

Availability of data Data are available from the corresponding author on reasonable request.
Authors' contributions All authors contributed to the study conception and design. Material preparation, data collection, and analysis were performed by all authors. The first draft of the manuscript was written by LP and ES, and all authors commented on previous versions of the manuscript. All authors read and approved the final manuscript.

Acknowledgements The authors would like to thank the staff of the Neurology and Geriatric units at the Careggi University Hospital of Florence and the General Medicine physicians for help in referring patients. AP acknowledges Fondazione Cassa di Risparmio di Firenze contribution for local support (Bando Ricerca Scientifica 2017).

Open Access This article is licensed under a Creative Commons Attribution-NonCommercial 4.0 International License, which permits any non-commercial use, sharing, adaptation, distribution and reproduction in any medium or format, as long as you give appropriate credit to the original author(s) and the source, provide a link to the Creative Commons licence, and indicate if changes were made. The images or other third party material in this article are included in the article's Creative Commons licence, unless indicated otherwise in a credit line to the material. If material is not included in the article's Creative Commons licence and your intended use is not permitted by statutory regulation or exceeds the permitted use, you will need to obtain permission directly from the copyright holder. To view a copy of this licence, visit http://creativecommons.org/licenses/by-nc/4.0/.

\section{References}

1. O’Brien JT, Erkinjuntti T, Reisberg B, et al. Vascular cognitive impairment. Lancet Neurol. 2003;2(2):89-98. https://doi.org/10. 1016/s1474-4422(03)00305-3. 
2. Pantoni L. Cerebral small vessel disease: from pathogenesis and clinical characteristics to therapeutic challenges. Lancet Neurol. 2010;9(7):689-701. https://doi.org/10.1016/S1474-4422(10) 70104-6.

3. Wardlaw JM, Smith EE, Biessels GJ, et al. Neuroimaging standards for research into small vessel disease and its contribution to ageing and neurodegeneration. Lancet Neurol. 2013;12(8):82238. https://doi.org/10.1016/S1474-4422(13)70124-8.

4. Pantoni L. Treatment of vascular dementia: evidence from trials with non-cholinergic drugs. J Neurol Sci. 2004;226(1-2):67-70. https://doi.org/10.1016/j.jns.2004.09.014.

5. Smith EE, Cieslak A, Barber P, et al. Therapeutic strategies and drug development for vascular cognitive impairment. J Am Heart Assoc. 2017;6(5):e005568. https://doi.org/10.1161/JAHA.117. 005568.

6. Selden NR, Gitelman DR, Salamon-Murayama N, Parrish TB, Mesulam MM. Trajectories of cholinergic pathways within the cerebral hemispheres of the human brain. Brain. 1998;121(Pt 12):2249-57. https://doi.org/10.1093/brain/121.12.2249.

7. Wilkinson D, Doody R, Helme R, et al. Donepezil in vascular dementia: a randomized, placebo-controlled study. Neurology. 2003;61(4):479-86. https://doi.org/10.1212/01.wnl.0000078943. 50032.fc.

8. Black S, Román GC, Geldmacher DS, et al. Efficacy and tolerability of donepezil in vascular dementia: positive results of a 24-week, multicenter, international, randomized, placebo-controlled clinical trial. Stroke. 2003;34(10):2323-30. https://doi. org/10.1161/01.STR.0000091396.95360.E1.

9. Dichgans M, Markus HS, Salloway S, et al. Donepezil in patients with subcortical vascular cognitive impairment: a randomised double-blind trial in CADASIL. Lancet Neurol. 2008;7(4):310-8. https://doi.org/10.1016/S1474-4422(08)70046-2.

10. Erkinjuntti T, Kurz A, Gauthier S, Bullock R, Lilienfeld S, Damaraju CV. Efficacy of galantamine in probable vascular dementia and Alzheimer's disease combined with cerebrovascular disease: a randomised trial. Lancet. 2002;359(9314):1283-90. https://doi.org/10.1016/S0140-6736(02)08267-3.

11. Auchus AP, Brashear HR, Salloway S, et al. Galantamine treatment of vascular dementia: a randomized trial. Neurology. 2007;69(5):448-58. https://doi.org/10.1212/01.wnl.0000266625. 31615.f6.

12. Ballard C, Sauter M, Scheltens P, et al. Efficacy, safety and tolerability of rivastigmine capsules in patients with probable vascular dementia: the VantagE study. Curr Med Res Opin. 2008;24(9):2561-74. https://doi.org/10.1185/030079908023281 42.

13. Narasimhalu K, Effendy S, Sim CH, et al. A randomized controlled trial of rivastigmine in patients with cognitive impairment no dementia because of cerebrovascular disease. Acta Neurol Scand. 2010;121(4):217-24. https://doi.org/10.1111/j.1600-0404. 2009.01263.x

14. Orgogozo JM, Rigaud AS, Stöffler A, Möbius HJ, Forette F. Efficacy and safety of memantine in patients with mild to moderate vascular dementia: a randomized, placebo-controlled trial (MMM 300). Stroke. 2002;33(7):1834-9. https://doi.org/10. 1161/01.str.0000020094.08790.49.

15. Wilcock G, Möbius HJ, Stöffler A, MMM 500 group. A doubleblind, placebo-controlled multicentre study of memantine in mild to moderate vascular dementia (MMM500). Int Clin Psychopharmacol. 2002;17(6):297-305. https://doi.org/10.1097/ 00004850-200211000-00005.

16. Kavirajan H, Schneider LS. Efficacy and adverse effects of cholinesterase inhibitors and memantine in vascular dementia: a meta-analysis of randomised controlled trials. Lancet Neurol. 2007;6(9):782-92. https://doi.org/10.1016/S1474-4422(07) 70195-3.
17. Gorelick PB, Scuteri A, Black SE, et al. Vascular contributions to cognitive impairment and dementia: a statement for healthcare professionals from the American Heart Association/American Stroke Association. Stroke. 2011;42(9):2672-713. https:// doi.org/10.1161/STR.0b013e3182299496.

18. Alvarez-Sabín J, Ortega G, Jacas C, et al. Long-term treatment with citicoline may improve poststroke vascular cognitive impairment. Cerebrovasc Dis. 2013;35(2):146-54. https://doi. org/10.1159/000346602.

19. Fioravanti M, Yanagi M. Cytidinediphosphocholine (CDP-choline) for cognitive and behavioural disturbances associated with chronic cerebral disorders in the elderly. Cochrane Database Syst Rev. 2005. https://doi.org/10.1002/14651858.CD000269. pub3.

20. Di Perri R, Coppola G, Ambrosio LA, Grasso A, Puca FM, Rizzo M. A multicentre trial to evaluate the efficacy and tolerability of alpha-glycerylphosphorylcholine versus cytosine diphosphocholine in patients with vascular dementia. J Int Med Res. 1991;19(4):330-41. https://doi.org/10.1177/030006059101900 406.

21. Muratorio A, Bonuccelli U, Nuti A, Battistini N, Passero S. A neurotropic approach to the treatment of multi-infarct dementia using L- $\alpha$-glyceryl-phoshorylcholine. Curr Ther Res. 1992;52(5):74151. https://doi.org/10.1016/S0011-393X(05)80518-1.

22. Pantoni L, Carosi M, Amigoni S, Mascalchi M, Inzitari D. A preliminary open trial with nimodipine in patients with cognitive impairment and leukoaraiosis. Clin Neuropharmacol. 1996;19(6):497-506. https://doi.org/10.1097/00002826-19961 9060-00003.

23. Pantoni L, Bianchi C, Beneke M, Inzitari D, Wallin A, Erkinjuntti T. The Scandinavian multi-infarct dementia trial: a double-blind, placebo-controlled trial on nimodipine in multi-infarct dementia. J Neurol Sci. 2000;175(2):116-23. https://doi.org/10.1016/s0022510x(00)00301-4.

24. Pantoni L, Rossi R, Inzitari D, et al. Efficacy and safety of nimodipine in subcortical vascular dementia: a subgroup analysis of the Scandinavian multi-infarct dementia trial. J Neurol Sci. 2000;175(2):124-34. https://doi.org/10.1016/s0022-510x(00) 00300-2.

25. Pantoni L, del Ser T, Soglian AG, et al. Efficacy and safety of nimodipine in subcortical vascular dementia: a randomized placebo-controlled trial. Stroke. 2005;36(3):619-24. https://doi.org/ 10.1161/01.STR.0000155686.73908.3e.

26. Farkas E, De Jong GI, Apró E, Keuker JI, Luiten PG. Calcium antagonists decrease capillary wall damage in aging hypertensive rat brain. Neurobiol Aging. 2001;22(2):299-309. https://doi.org/ 10.1016/s0197-4580(00)00225-6.

27. de Jong GI, Traber J, Luiten PG. Formation of cerebrovascular anomalies in the ageing rat is delayed by chronic nimodipine application. Mech Ageing Dev. 1992;64(3):255-72. https://doi. org/10.1016/0047-6374(92)90083-p.

28. Hu M, Liu Z, Lv P, et al. Nimodipine activates neuroprotective signaling events and inactivates autophages in the VCID rat hippocampus. Neurol Res. 2017;39(10):904-9. https://doi.org/10. 1080/01616412.2017.1356157.

29. López-Arrieta JM, Birks J. Nimodipine for primary degenerative, mixed and vascular dementia. Cochrane Database Syst Rev. 2002. https://doi.org/10.1002/14651858.CD000147.

30. Salvadori E, Poggesi A, Donnini I, et al. Association of nimodipine and choline alphoscerate in the treatment of cognitive impairment in patients with cerebral small vessel disease: study protocol for a randomized placebo-controlled trial-the CONIVaD trial. Aging Clin Exp Res. 2020;32(3):449-57. https://doi.org/10.1007/ s40520-019-01229-Z. 
31. Morris JC. The Clinical Dementia Rating (CDR): current version and scoring rules. Neurology. 1993;43(11):2412-4. https://doi. org/10.1212/wnl.43.11.2412-a.

32. Pantoni L, Basile AM, Pracucci G, et al. Impact of age-related cerebral white matter changes on the transition to disability-the LADIS study: rationale, design and methodology. Neuroepidemiology. 2005;24(1-2):51-62. https://doi.org/10.1159/000081050.

33. van Swieten JC, Hijdra A, Koudstaal PJ, van Gijn J. Grading white matter lesions on CT and MRI: a simple scale. J Neurol Neurosurg Psychiatry. 1990;53(12):1080-3. https://doi.org/10.1136/jnnp.53. 12.1080 .

34. McKhann GM, Knopman DS, Chertkow H, et al. The diagnosis of dementia due to Alzheimer's disease: recommendations from the National Institute on Aging-Alzheimer's Association workgroups on diagnostic guidelines for Alzheimer's disease. Alzheimers Dement. 2011;7(3):263-9. https://doi.org/10.1016/j.jalz.2011. 03.005 .

35. American Psychiatric Association. Diagnostic and statistical manual of mental disorders. 5th ed. Arlington: American Psychiatric Publishing; 2013.

36. Nasreddine ZS, Phillips NA, Bédirian V, et al. The Montreal Cognitive Assessment, MoCA: a brief screening tool for mild cognitive impairment. J Am Geriatr Soc. 2005;53(4):695-9. https://doi. org/10.1111/j.1532-5415.2005.53221.x (Published correction appears in J Am Geriatr Soc. 2019 Sep;67(9):1991).

37. Katz S, Ford AB, Moskowitz RW, Jackson BA, Jaffe MW. Studies of illness in the aged. The index of ADL: a standardized measure of biological and psychosocial function. JAMA. 1963;185:914-9. https://doi.org/10.1001/jama.1963.03060120024016.

38. Lawton MP, Brody EM. Assessment of older people: self-maintaining and instrumental activities of daily living. Gerontologist. 1969;9(3):179-86.
39. Gélinas I, Gauthier L, McIntyre M, Gauthier S. Development of a functional measure for persons with Alzheimer's disease: the disability assessment for dementia. Am J Occup Ther. 1999;53(5):471-81. https://doi.org/10.5014/ajot.53.5.471.

40. van Straten A, de Haan RJ, Limburg M, Schuling J, Bossuyt PM, van den Bos GA. A stroke-adapted 30-item version of the sickness impact profile to assess quality of life (SA-SIP30). Stroke. 1997;28(11):2155-61. https://doi.org/10.1161/01.str.28.11.2155.

41. Radloff LS. The CES-D scale: a self-report depression scale for research in the general population. Appl Psychol Meas. 1977;1(3):385-401. https://doi.org/10.1177/014662167700100 306.

42. Salvadori E, Poggesi A, Pracucci G, Inzitari D, Pantoni L, VMCITuscany Study Group. Development and psychometric properties of a neuropsychological battery for mild cognitive impairment with small vessel disease: the VMCI-Tuscany Study. J Alzheimers Dis. 2015;43(4):1313-23. https://doi.org/10.3233/JAD-141449.

43. Strom BL, Kimmel SE, Hennessy S. Pharmacoepidemiology. New York City: Wiley; 2019.

44. Poggesi A, Salvadori E, Pantoni L, et al. Risk and determinants of dementia in patients with mild cognitive impairment and brain subcortical vascular changes: a study of clinical, neuroimaging, and biological markers-the VMCI-Tuscany Study: rationale, design, and methodology. Int J Alzheimers Dis. 2012;2012:608013. https://doi.org/10.1155/2012/608013.

45. The LADIS Study Group, Poggesi A, Pantoni L, Inzitari D, et al. 2001-2011: a decade of the LADIS (Leukoaraiosis And DISability) study: what have we learned about white matter changes and small-vessel disease? Cerebrovasc Dis. 2011;32(6):577-88. https://doi.org/10.1159/000334498.

\section{Authors and Affiliations}

\section{Emilia Salvadori ${ }^{1,2} \cdot$ Anna Poggesi $^{1,2} \cdot$ Ida Donnini $^{1} \cdot$ Valentina Rinnoci $^{1} \cdot$ Guido Chiti $^{2,3} \cdot$ Martina Squitieri ${ }^{2,4}$. Laura Tudisco $^{2} \cdot$ Fabio Fierini $^{5} \cdot$ Anna Melone $^{2} \cdot$ Francesca Pescini $^{6} \cdot$ Leonardo Pantoni $^{7}$ (1)}

1 IRCCS Fondazione Don Carlo Gnocchi, Florence, Italy

2 NEUROFARBA Department, University of Florence, Florence, Italy

3 Present Address: SOC Neurologia, AUSL Toscana Centro, Ospedale Santo Stefano, Prato, Italy

4 Present Address: SOC Neurologia, AUSL Toscana Centro, Ospedale San Giovanni di Dio, Firenze, Italy
5 Psychiatry Unit, Department of Health Sciences, University of Florence, Florence, Italy

6 Stroke Unit, Emergency Department, Azienda Ospedaliero Universitaria Careggi, Florence, Italy

7 Department of Biomedical and Clinical Sciences, 'L. Sacco' Hospital, University of Milan, Via Giovanni Battista Grassi 74, 20157 Milan, Italy 